\title{
An inter-professional education programme for diabetes care in London
}

\author{
Daniel L Ching, Kenneth A Earle
}

\begin{abstract}
Over the years there has been a steady increase in avoidable referrals from primary to secondary care for newly diagnosed diabetic patients. Audits have shown that diabetes referral rates were rising yearly. Secondary care is becoming overwhelmed with the heavy workload and increasing cost, which also led to compromising care for complex patients. This led to the design and implementation of a diabetes based interprofessional education (IPE) programme.

The IPE programme was taught in cycles. Each cycle consists of 10 sessions. One session was taught for one afternoon a week over 10 weeks. On the 11th week an OSCE style end of course assessment was performed. Health care professionals (HCPs) from different professions were taught in the same classroom, using the same material.

A re-audit of diabetes referral rates showed a change in referral ratio post-programme. Qualitative interviews using Kirkpatrick and Barr's hierarchy were performed 2 years post-programme to assess learners' outcomes. Results show that the effects of the programme were sustained beyond 2 years and that these changes were carried into practice. There was a change in HCPs attitude and perception and more importantly it showed improvement in patient outcomes. This represents a novel IPE programme for diabetes care which has shown to be able to increase confidence, capacity and scope of care provided by HCPs in the community.
\end{abstract}

\section{Problem}

Audits of diabetes referral rates in this London borough have shown that over the years there has been a steady increase in avoidable referral rates from primary care to secondary care for newly diagnosed diabetic patients. Secondary care is becoming overwhelmed with the heavy workload and increasing cost, which also led to compromising care for complex patients. Based on projected population change and trends in obesity, prevalence of diabetes in London borough of Wandsworth is set to increase by $15.4 \%$ from 2010-2025 unless successful preventative measures are implemented (1). This led to the design and implementation of a diabetes based inter-professional education (IPE) programme.

\section{Background}

The need for integration of services to improve quality and efficiency in healthcare is inevitable. For integration to be successful, collaboration is one of the fundamental principles. There are various types of interventions to increase collaboration and interprofessional education (IPE) is one of them. Complexities of diabetes mean that management requires an integrated pathway of services. Diabetes is a significant health problem in the UK with the current prevalence estimated to be $5.5 \%$ in adults (2).

\section{Baseline measurement}

Diabetes referral rates were obtained through secondary care referral database and diabetes registers of GP practices. Data was audited before the IPE programme intervention and 15 months after. Pre-programme new-referral cases were 1793 and follow-up cases were 5155 (Figure 1).

\section{Design}

The IPE program called the Wandsworth Rolling Initiative in Training and Education (WRiiTE) was created to increase the knowledge of healthcare professionals (HCPs) in diabetes and promote further collaboration within the community. The overall aim of the programme was to equip primary care to deal with more complex cases, reducing straightforward referrals to secondary care in accordance with the referral document, and thus freeing up resources in secondary care to deal with complicated cases. Having appropriate cases dealt with at appropriate levels will increase efficiency and effectiveness of the whole system. This is expected to provide quality improvement in diabetes care and reduce patient time spent in healthcare.

This programme was developed jointly between allied HCPs from the diabetes unit at St Georges Healthcare NHS Trust. The programme was designed to involve consultants, diabetes specialist nurses, community diabetes specialist nurses, chiropodists, dieticians, GP's, nurse practitioners, and practice nurses.

\section{Strategy}

This programme was taught for one afternoon a week over 10 weeks. All topics were based on diabetes guidelines and were selected according to results from a needs assessment formed prior the sessions. Each session included lectures and workshops. On 
week 11, a formative OSCE style end of course assessment (OPERA) was performed. HCPs from different professions were taught in the same classroom using the same material. This provided an opportunity to learn the same material at the same rate with discussions from other professions perspective.

Ideally, one GP and one PN from each surgery were encouraged to attend the same cycle. It was hoped that through attending the course together they would be able to use the time to understand each other better. However, in practice this was challenging as GP surgeries are often busy and having two members away for the same afternoon was difficult.

A subcomponent of the programme was the reach-out clinics, where consultants would visit primary care practices to observe consultations with complex patients. Involvement in this component was voluntary and not all practices chose to participate.

A set of local referral guidelines was produced to assist in referrals. A project manager was hired and there was collaboration with NHS Wandsworth to facilitate the organisation of the programme.

\section{Results}

Qualitative interviews were performed with key stakeholders two years after the programme to assess the learner's outcome (Table 1). All interviews were performed using semi-structured, face-toface interviews. A semi-structured format was preferred because it allowed information obtained from the interviews to be relevant to the research objectives and at the same time giving interviewees flexibility to raise issues and generate a wide range of information.

The sampling method used was a non-probability, purposive sampling with a goal of maximum variation of categories. This was chosen to ensure contribution from all stakeholders in every area, and was necessary to gain different perspectives. A total of eight primary care HCPs were interviewed. They were from three professions - general practitioners, practice nurses, and nurse practitioners.

Interview questions were composed according to pre-identified themes discovered through literature review and issues that were thought-provoking. A framework analysis method using Kirkpatrick \& Barr's learner outcome hierarchy was used for analysis of the interview data (3).

All interviewees acknowledged that learning with different professions in the same classroom was beneficial to them. The learning made them appreciate each other's skills and roles and develop a sense of respect for each other. It also made them aware who to consult when a problem occurs.

The nature of diabetes as a constantly developing condition requires the need for all HCPs to be updated regularly. From the interviews it was not apparent that there were any differences in knowledge between GPs and PNs that prevented them from learning together. Although GPs tend to regard themselves of higher status, nurses may be more familiar in diabetes management through their practical experience.

All interviewees became more confident in diabetes management after the course. This confidence appears to have filtered into patient care. Interviewees felt the programme was effective in improving patient outcomes as they were improving over time. One interviewee mentioned that the impact of the course was sustained since it appears that knowledge and confidence gained during the course was internalised into daily practice and has become a routine part of care. On most occasions, interviewees appear to know what they are doing, and the course was useful in reassuring them that their current practice was correct.

Post-programme new-referral rates decrease to 1171 and follow-up cases increase to 5849 (Figure 1). Referral ratio changes suggest it could be due to primary care's increase ability to deal with uncomplicated diabetes cases which frees the resources in secondary care to deal with complicated cases. This was supported by interviews indicating that majority of referrals were of a more complex nature.

Results show that effects of programme were sustained beyond 2 years and these changes were carried into practice. There was a change in HCPs attitude and perception and more importantly it has shown improvement in patient outcomes.

See supplementary file: ds1915.docx - "Results"

\section{Lessons and limitations}

This model is associated with improvement of diabetes quality measures. It is also successful in achieving its aim of up-skilling competence of HCPs in primary care and increasing their scope of practice for patients with diabetes. Improved collaboration between primary and secondary care creates synergy and improves utilisation of resources. The course content was relevant and applicable for the new service redesign encompassed within the Health and Social Care Bill.

A major limitation of this evaluation is that there was no control group for comparison. There may be various confounding factors affecting the reliability and validity of the results and future research will involve designing an evaluation in a controlled environment.

\section{Conclusion}

After this programme, the HCPs in secondary care considered that referrals were more appropriate. With increased knowledge and confidence, primary care is now more aggressive with treatment, keeping diabetic quality measures within the desired range for longer periods. More patients are now seen locally, which will increase their convenience and reduce travelling time. The increase in collaboration between primary and secondary care will lead to more holistic and patient-centred care. This novel IPE programme for diabetes care has been shown to be able to increase confidence, capacity and scope of care provided by HCPs in the community. 


\section{References}

1. Wandsworth PCT Department of Public Health. Diabetes Health Needs Assessment: NHS Wandsworth. 2010.

2. Diabetes UK. Diabetes in the UK 2012: Key statistics on diabetes. April 2012.

3. Freeth D., Reeves S., Koppel I., Hammick M., Barr H. Evaluating interprofesisonal education: A self help guide 2005.

\section{Declaration of interests}

Nothing to declare

\section{Acknowledgements}

Oliver Groene 